\title{
Theaflavin attenuates cerebral ischemia/reperfusion injury by abolishing miRNA-128-3p-mediated Nrf2 inhibition and reducing oxidative stress
}

\author{
RONGGANG LI ${ }^{1,2}, \mathrm{XIN} \mathrm{LI}^{3}, \mathrm{HAIBING} \mathrm{WU}^{4}, \mathrm{ZHIKUN} \mathrm{YANG}{ }^{2}, \mathrm{LI} \mathrm{FEI}^{2}$ and JIANHONG ZHU ${ }^{1}$ \\ ${ }^{1}$ Department of Neurosurgery, Fudan University Huashan Hospital and State Key Laboratory of Medical Neurobiology, \\ Institutes of Brain Science, Shanghai Medical College, Fudan University, Shanghai 200040; Departments of ${ }^{2}$ Neurosurgery \\ and ${ }^{3}$ Imaging, Jinshan Hospital Affiliated to Fudan University; ${ }^{4}$ Shanghai Public Health Clinical Center, \\ Fudan University, Shanghai 201508, P.R. China
}

Received March 21, 2019; Accepted August 23, 2019

DOI: $10.3892 / \mathrm{mmr} .2019 .10755$

\begin{abstract}
Theaflavin has been proven to own strong antioxidative capacity; however, the molecular mechanism underlying its protective effect against cerebral ischemia-reperfusion $(\mathrm{I} / \mathrm{R})$ injury remains unclear. Therefore, the present study was designed to elucidate the neuroprotective effects of theaflavin on cerebral I/R injury and its underlying molecular mechanisms. To investigate the effects of theaflavin on neurological function, neurogenesis, and oxidative stress, experiments were performed using a cerebral I/R injury rat model, and neural stem cells (NSCs) were subjected to oxygen-glucose deprivation and reoxygenation (OGD/R). Further, the expression profiles of miRNA-128-3p and the regulatory function of nuclear factor (erythroid-derived 2)-related factor 2 (Nrf2) were evaluated in these models. We found that theaflavin treatment significantly reduced infarct volume and neuronal injury, and thus improved the impaired memory and learning ability. Furthermore, theaflavin treatment significantly enhanced the increase in NSC proliferation, reduction in the apoptotic rate and inhibition of oxidative stress. Mechanistically, theaflavin targeted miRNA-128-3p and further activated the Nrf2 pathway to reduce oxidative stress. In summary, theaflavin has a strong ability to attenuate cerebral I/R injury through
\end{abstract}

Correspondence to: Dr Li Fei, Department of Neurosurgery, Jinshan Hospital Affiliated to Fudan University, 1508 Long Hang Road, Shanghai 201508, P.R. China

E-mail: 18930819998@189.cn

Dr Jianhong Zhu, Department of Neurosurgery, Fudan University Huashan Hospital and State Key Laboratory of Medical Neurobiology, Institutes of Brain Science, Shanghai Medical College, Fudan University, 12 Urumqi Mid Road, Shanghai 200040, P.R. China

E-mail: jzhu@fudan.edu.cn

Key words: theaflavin, cerebral ischemia/reperfusion injury, neural stem cells, oxidative stress, miRNA-128-3p, Nrf2
miRNA-128-3p-mediated recovery of the impaired antioxidant defense system, which suggests that it could be a potential drug candidate for ischemic stroke.

\section{Introduction}

Cerebrovascular disease is a common disease with high morbidity and mortality worldwide (1). Ischemic stroke as a type of cerebrovascular disease can lead to cognitive impairment and neurological deficit (2). At present, thrombolysis, which effectively reduces infarct size, is the main treatment for ischemic stroke (3). However, cerebral ischemia/reperfusion (I/R) injury often occurs irreversibly in ischemic brain tissue after restoration of blood perfusion, which severely restricts the recovery of patients with ischemic stroke (4). Although some promising neuroprotective agents have been identified, such as curcumin, rosmarinic acid, astragaloside VI and berberine $(5,6)$, their protective effects are still limited. Therefore, identification of novel neuroprotective agents for cerebral I/R injury is highly desired.

In recent years, abundant evidence shows that oxidative stress plays an important role in the pathogenesis of cerebral I/R injury (7). Wu et al found that reactive oxygen species (ROS) and malondialdehyde (MDA) were significantly increased in rats with middle cerebral artery occlusion (MCAO), whereas the activity of antioxidants was markedly reduced (7). The impaired antioxidant defense system was found to lead to oxidative damage of brain lipids, proteins and DNA, further leading to cell death and brain dysfunction $(8,9)$. Nuclear factor (erythroid-derived 2)-related factor 2 (Nrf2) is a key transcription factor that regulates the antioxidant stress response (10). Many studies have shown that activation of the Nrf2 pathway can protect against cerebral I/R injury in vivo and in vitro $(11,12)$. Neuroprotective agents, such as resveratrol, isoquercetin and protocatechualdehyde also reportedly protect the brain from cerebral I/R injury through Nrf2 activation $(9,11,13)$. Therefore, repair of the endogenous antioxidant system through activation of the Nrf2 pathway may be an effective strategy to alleviate cerebral I/R injury. 
Many studies have demonstrated that plant extracts are potential neuroprotective agents in the treatment of ischemic stroke (14). Theaflavin, which is derived from black tea, has been widely used as an antioxidant. Feng et al reported that theaflavin prevents DNA damage by suppressing oxidative stress and inhibiting cytochrome P450 1A1 in vitro (15). In addition, theaflavin demonstrated neuroprotective effects by inhibiting the activity of oxidant enzymes in PC12 cells (16). These findings provide the foundation for a new field of exploration using theaflavins in the treatment of cerebrovascular disease caused by oxidative stress. Cai et al reported that theaflavin ameliorates cerebral I/R injury in rats through anti-inflammatory effects and modulation of signal transducer and activator of transcription (STAT)-1 (17). However, the exact molecular mechanism by which theaflavin attenuates cerebral I/R injury through antioxidative stress still awaits further investigation.

The non-regeneration and vulnerability of nerves suggest that they are extremely fragile and difficult to repair once damaged. Endogenous neural stem cells (NSCs) with proliferative ability exist in at least two regions of the central nervous system of adult mammals, the hippocampus and lateral ventricle $(18,19)$. NSCs can self-renew, generate neuroblasts and migrate to damaged areas of the brain to replace dead nerve cells to induce neurological activity $(20,21)$. Because of the relatively small number of NSCs, their proliferation is essential for the recovery of neural function and repair of cognitive impairment in cerebral I/R injury. Therefore, exploration of the effects of neuroprotective agents on NSC proliferation has considerable clinical significance.

Herein, an in vivo experimental animal model of stroke and in vitro model of NSCs that were subjected to oxygen-glucose deprivation and reoxygenation (OGD/R) were used to demonstrate that theaflavin is able to promote neurogenesis and repair neurological function by inhibiting oxidative stress to induce brain recovery.

\section{Materials and methods}

Cerebral I/R model (7). In total, 50 Male Sprague-Dawley (SD) rats, 7-8 weeks old (body weight 270-300 g) were obtained from East China Normal University [Production License: SCXK (Shanghai) 2016-0004]. All animal protocols were approved and regulated by the Animal Care and Ethics Committee of East China Normal University. All rats were allowed free access to water and food and maintained at a controlled temperature range $\left(20 \pm 2^{\circ} \mathrm{C}\right)$. The intraluminal suture middle cerebral artery occlusion (MCAO) method was performed to induce focal cerebral ischemia. Rats were anesthetized with isoflurane (4\%) in $\mathrm{N}_{2} \mathrm{O}$ gas $(70 \%)$ with $\mathrm{O}_{2}(30 \%)$ in all experiments. A midline cervical incision was used to expose the left-sided carotid arteries. The left external carotid artery was dissected and isolated distally by coagulating its branches, on which a distal ligature was placed. A piece of 3-0 tip rounded monofilament nylon suture was introduced via the lumen of the left external/internal carotid artery stump to be embedded into the left anterior cerebral artery. At $1.5 \mathrm{~h}$ of cerebral ischemia, the monofilament nylon suture was withdrawn to permit reperfusion. During the surgical process, moorVMS-LDF1-HP high power laser doppler detector
(Jiander Technology Co., Ltd., Beijing, China) was used to monitor blood flow changes. An abrupt reduction in regional cerebral perfusion to less than $30 \%$ of the baseline value was considered to achieve focal ischemia. For the sham group, the surgical process was the same as that for the MCAO group but without suture occlusion.

Primary cultured NSCs. Primary cultured NSCs were harvested from the hippocampus in embryonic SD rats aged 14-16 days as previously described (21). In brief, the fore cortex in rat embryos was isolated and ground into single cells using nylon mesh $(70 \mu \mathrm{m})$. Cells were then re-suspended in Dulbecco's modified Eagle's medium (DMEM)/Nutrient Mixture F-12 (DMEM/F12) (Invitrogen, Thermo Fisher Scientific, Inc., Waltham, MA, USA) and were plated in $10-\mathrm{cm}$ dishes for culture at $1 \times 10^{4}$ cells $/ \mathrm{cm}^{2}$ in DMEM/F12 medium with fetal bovine serum (FBS, 10\%; Gibco, Thermo Fisher Scientific, Inc.), epidermal growth factor (EGF, $10 \mathrm{ng} / \mathrm{ml}$; Sigma-Aldrich; Merck KGaA, Darmstadt, Germany), B27 (2\%; Invitrogen; Thermo Fisher Scientific, Inc.), basic fibroblast growth factor (bFGF, $10 \mathrm{ng} / \mathrm{ml}$; Sigma-Aldrich; Merck KGaA), penicillin (5 IU; Sigma-Aldrich; Merck KGaA), and streptomycin (5 $\mu \mathrm{g} / \mathrm{ml}$; Sigma-Aldrich; Merck KGaA). Cells were incubated in a humidified atmosphere containing $21 \% \mathrm{O}_{2}$ and $78 \% \mathrm{~N}_{2}$ supplied with $5 \% \mathrm{CO}_{2}$ at $37^{\circ} \mathrm{C}$ for 5 days to form a neurosphere (diameter $\sim 100 \mu \mathrm{m}$ ). The neurospheres were collected and triturated to a single-NSC suspension. The NSCs were then replanted at $1 \times 10^{4}$ cells $/ \mathrm{cm}^{2}$ and passaged every 4 to 6 days. These NSCs still possessed self-renewal, proliferation and differentiation capacity until the 10th passage. The NSCs of the 2nd to 5 th passages were used for subsequent experiments. To establish the OGD/R cellular model, NSCs were cultured in glucose-free balanced salt solution under hypoxic conditions $\left(1 \% \mathrm{O}_{2}, 5 \% \mathrm{CO}_{2}\right.$, and $\left.94 \% \mathrm{~N}_{2}\right)$ at $37^{\circ} \mathrm{C}$ for $3 \mathrm{~h}$. Subsequently, NSCs were cultured in normal medium and normoxic conditions $\left(21 \% \mathrm{O}_{2}, 5 \% \mathrm{CO}_{2}\right.$ and $\left.78 \% \mathrm{~N}_{2}\right)$.

Theaflavin treatments. Theaflavin [98.0\%, high-performance liquid chromatography (HPLC) grade] was purchased from Shyuanye Biotechnology (Shanghai, China). In the in vivo animal study, theaflavin dissolved in saline was intravenously injected into SD rats (10 mg/ $\mathrm{kg}$ group and $50 \mathrm{mg} / \mathrm{kg}$ group) $2 \mathrm{~h}$ after MCAO ischemia, once a day for 7 days. For the $10 \mathrm{mg} / \mathrm{kg}$ group, SD rats received theaflavin at $10 \mathrm{mg} / \mathrm{kg}$; for the $50 \mathrm{mg} / \mathrm{kg}$ group, SD rats received theaflavin at $50 \mathrm{mg} / \mathrm{kg}$. The same volume of saline was injected into SD rats (MCAO group). For in vitro experiments, theaflavin dissolved in phosphate-buffered saline (PBS) was added to the DMEM/F12 medium. The NSCs were seeded into 6/96-well plates in the DMEM/F12 media with theaflavin. After incubation for $2 \mathrm{~h}$, NSCs were challenged with OGD/R. The NSCs were divided into the following: Control group, OGD/R group (same volume of PBS), $2 \mu \mathrm{M}$ theaflavin group, and $10 \mu \mathrm{M}$ theaflavin group. After incubation for $48 \mathrm{~h}$, a series of following experiments were conducted.

Tissue preparation and staining. The SD rats were sacrificed at 7 days after theaflavin treatment. The harvested brain tissues were cut into five equally-sized coronal sections (2-3 mm thickness) using an EM UC7 microtome (Leica 
Microsystems Inc., Wetzlar, Germany). The brain sections were then incubated with $2 \%$ 2,3,5-triphenyltetrazolium chloride (TTC; Sigma-Aldrich; Merck KGaA) at $37^{\circ} \mathrm{C}$ for 15 min with gentle shaking. Subsequently, the brain sections were fixed with paraformaldehyde (4\%) overnight. The stained slices were photographed and the infarct size was quantified using the Image-Pro Plus 6.0 software (Media Cybernetics, Inc., Rockville, MD, USA). For terminal deoxynucleotidyl transferase dUTP nick end labeling (TUNEL) staining (Beyotime Institute of Biotechnology, Haimen, China), the harvested brain tissues were cut into $2.5 \mathrm{~mm}$ blocks and fixed with paraformaldehyde overnight. Subsequently, blocks were embedded in paraffin and cut into 4- $\mu \mathrm{m}$ coronal sections, according to the manufacturer's protocol. Apoptotic images were obtained using the Axioplan 2 imaging system (Carl Zeiss AG, Oberkochen, Germany). Six sections of each sample were selected and five fields of each section were examined for quantification of apoptosis.

Evaluation of neurological deficits, learning and memory ability. Neurological deficit testing in each group was conducted by an investigator who was blinded to the experimental groups according to the method of Longa et al (22). Neurological findings were scored on a five-point scale: Depressed levels of consciousness and inability to walk spontaneously $=4$; falling to the right $=3$; circling to the right $=2$; failure to extend right forepaw fully $=1$; and no neurological deficits $=0$. The Morris water maze test $(9,11)$ was conducted to evaluate memory and learning function by an investigator blinded to the experimental groups. To make the water opaque, non-toxic ink was added. Rats were brought into the conducting room $30 \mathrm{~min}$ prior to the test. They were subjected to four trials per day for 5 consecutive days. Rats were given $1 \mathrm{~min}$ to find the platform in each trial. The escape latency was recorded when experimental rats reached the platform. It was recorded as $1 \mathrm{~min}$ even when the rat could not reach the platform within $1 \mathrm{~min}$. Rats were allowed to stay on the platform for another $15 \mathrm{sec}$ after locating it. After the test, rats were dried and then returned to their cages. A 4-min inter-trial rest was allowed. The probe trial was performed on the sixth day. The round tank was divided into four equal quadrants. The target circle was considered as an area $20 \mathrm{~cm}$ in diameter, as measured from the platform center. The platform crossing frequency was defined as the number of times the rat crossed the area within $1.5 \mathrm{~min}$.

Neurosphere assay (6). Primary cultured NSCs that were subjected to both theaflavin treatment and $\mathrm{OGD} / \mathrm{R}$ were fixed with paraformaldehyde (4\%) for $20 \mathrm{~min}$. After being rinsed with PBS, NSCs were incubated with blocking buffer (PBS, $0.1 \%$ Triton-100, and $100 \%$ goat serum) for $2 \mathrm{~h}$. The primary antibody against Nestin (mouse, dilution 1:300; Abcam, Cambridge, UK; cat. no. ab6142) was added at $4^{\circ} \mathrm{C}$ overnight. After being washed with PBS, the secondary antibody (Alexa 568 goat anti-mouse, dilution 1:300; Huabio; cat. no. HA1017) was added at $25^{\circ} \mathrm{C}$ in the dark for $2 \mathrm{~h}$. Subsequently, NSCs were incubated with 4',6-diamidino-2-phenylindole (DAPI) for $10 \mathrm{~min}$ to identify the neurosphere, after which they were washed with PBS. The neurosphere images were obtained using a Zeiss Axioskop 50 Trinocular Fluorescence
Microscope (Carl Zeiss; magnification, x200). Fifteen fields were randomly chosen in each well. The diameter of the neurosphere was quantified using the Image-Pro Plus 6.0 software (Media Cybernetic).

Cell Counting Kit-8 (CCK-8) assay and flow cytometry. Cell viability of NSCs was evaluated using the CCK-8 assay (Sigma-Aldrich; Merck KGaA). Primary cultured NSCs (1x10 $/$ well) were seeded into 96-well plates and cultured for $24 \mathrm{~h}$. The NSCs were then subjected to theaflavin treatment and OGD/R. Subsequently, the NSCs were incubated with $10 \%$ water-soluble tetrazolium dye (WST-8). The optical density (OD) was then measured using a LUX microplate reader (Thermo Fisher Scientific) at an absorbance of $450 \mathrm{~nm}$. The apoptotic rate in NSCs was detected using the Annexin V-FITC Apoptosis kit (Beyotime) according to the manufacturer's instructions. Primary cultured NSCs were seeded into 6-well plates and cultured for $24 \mathrm{~h}$. The NSCs were then subjected to theaflavin treatment and OGD/R. Subsequently, the NSCs were incubated with Annexin $\mathrm{V}$ and then added to propidium iodide (PI) buffer for $15 \mathrm{~min}$ at $25^{\circ} \mathrm{C}$ in a dark room. The apoptotic cells were quantified by a FACSAria II flow cytometer (BD Biosciences, Franklin Lakes, NJ, USA).

Detection of oxidative stress. The supernatant containing the lysates of rat brain tissues and NSCs was homogenized in ice-cold mitochondria isolation buffer. The total ROS level in the brain tissue was detected using the Total ROS Activity Assay kit (Sigma-Aldrich; Merck KGaA). The supernatant lysate was incubated with Total ROS Green in an incubator $\left(5 \% \mathrm{CO}_{2}\right)$ for $6 \mathrm{~h}$ at $37^{\circ} \mathrm{C}$. Fluorescence intensity was then detected at $525 \mathrm{~nm}$ emission and $490 \mathrm{~nm}$ excitation. The levels of malondialdehyde (MDA), glutathione peroxidase (GSH-Px), and superoxide dismutase (SOD) in the rat brain tissues and NSCs were measured using standard assay kits, including the Total SOD Activity Detection kit (Solarbio, Beijing, China), Malondialdehyde (MDA) Content Detection kit (Solarbio), and Glutathione Peroxidase (GSH-Px) Detection kit (R\&D Systems, Inc., Minneapolis, MN, USA), according to the manufacturer's instructions. The OD value was then measured using a LUX microplate reader (Thermo Fisher Scientific).

RNA extraction and quantitative real-time PCR (qRT-PCR). According to the manufacturer's instructions, total RNA was extracted and complementary DNA (cDNA) was synthesized using the Animal Total RNA Isolation kit (Sangon Biotech Co., Ltd., Shanghai, China) and M-MuLV First Strand cDNA Synthesis kit (Sangon Biotech Co., Ltd.). qRT-PCR was performed using SYBR Real-Time PCR kit (Genepharma, Shanghai, China) under the following conditions: $95^{\circ} \mathrm{C}$ for $600 \mathrm{sec}$; followed by 45 cycles at $95^{\circ} \mathrm{C}$ for $10 \mathrm{sec} ; 60^{\circ} \mathrm{C}$ for $20 \mathrm{sec}$; and $72^{\circ} \mathrm{C}$ for $30 \mathrm{sec}$. The relative fold expression was calculated using the $2^{-\Delta \Delta \mathrm{Cq}}$ method (23). Each analysis was performed in triplicate. In addition, $U 6$ served as an internal reference gene for miRNAs, and $\beta$-actin, for mRNA. The sequences were as follows: miRNA-128-3p forward, 5'-GGT CACAGTGAACCGGTC-3' and reverse, 5'-GTGCAGGGT CCGAGGT-3'; U6 forward, 5'-CTCGCTTCGGCAGCA CA-3' and reverse, 5'-AACGCTTCACGAATTTGCGT-3'; Nrf2 forward, 5'-AGCACACCCAGTCAGAAACCAG-3' 
and reverse, 5'-TCTACAAACGGGAATGTCG-3'; $\beta$-actin forward, 5'-GGTCACAGTGAACCGGTC-3' and reverse, 5'-GTGCAGGGT CCGAGGT-3'.

Cell transfection. For cell transfection, NSCs were seeded at a density of $5.5 \times 10^{5} /$ well in a 6 -well plate $12 \mathrm{~h}$ before transfection. For miRNA-128-3p downregulation, NSCs were transfected with $100 \mathrm{nM}$ negative control miRNA 5'-CAG UACUUUUGUGUAGUACAAA-3' (NC miRNA) or $100 \mathrm{nM}$ miRNA-inhibitor 5'-AAAGAGACCGGUUCACUGUGA-3 (GenePharma, Shanghai, China) using Lipofectamine 2000 (Invitrogen; Thermo Fisher Scientific), according to the manufacturer's instructions, and incubated for an additional 48 h. For miRNA-128-3p upregulation, NSCs were transfected with $100 \mathrm{nM}$ negative control miRNA (NC miRNA) sense: 5'-UUCUCCGAACGUGUCACGUTT-3' and antisense: 5'-ACGUGACACGUUCGGAGAATT-3' or $100 \mathrm{nM}$ miRNA-mimics, sense: 5'-UCACAGUGAACCGGUCUC UUUAGU-3' and antisense: 5'-UAAAGAGACCGGUUC ACUGUGAUU-3' (GenePharma, Shanghai, China) using Lipofectamine 2000 (Invitrogen; Thermo Fisher Scientific), according to the manufacturer's instructions, and incubated for an additional $48 \mathrm{~h}$. After transfection, the NSCs were harvested for further analysis. Lentivirus-mediated Nrf2 plasmids (OE-Nrf2) were constructed by GenePharma Co. For Nrf2 overexpression, cells were transfected with lentivirus-mediated Nrf2 plasmids. Cells transfected with an empty vector were also employed and investigated. The transfected cells were subsequently harvested for further analysis.

Dual-luciferase reporter assay. The 3'-untranslated region (3'UTR) of Nrf2 was amplified by Sangon, and the sequences were inserted into a pMIR-reporter luciferase vector (Ambion, Rockville, MD, USA). The mutated 3'UTR was designed, constructed, and then inserted into the luciferase vector. The mutated-type (MUT) and wild-type (WT) 3'UTR vectors, NC miRNA, and miRNA mimics were co-transfected into 293 cells, alternately. The Dual-Luciferase Reporter Assay (Activemotif, USA) was performed to measure luciferase intensity 2 days after co-transfection.

Western blot analysis. Rat brain tissues and NSCs were harvested using a lysis buffer solution [radioimmunoprecipitation assay (RIPA) buffer, Beyotime Biotechnology], protease inhibitor cocktail (Thermo Fisher Scientific), and phosphatase inhibitor cocktail (Beyotime Biotechnology). After protein quantification using the bicinchoninic acid (BCA) protein concentration assay kit (Thermo Fisher Scientific), Total protein $(50 \mu \mathrm{g})$ was separated by $10 \%$ sodium dodecyl sulfate polyacrylamide gel electrophoresis (SDS-PAGE). After being transferred onto a polyvinylidene difluoride (PVDF) membrane, the protein was probed with primary antibodies for Nrf2 (rat, dilution 1:300; Abcam; cat. no. ab137550) and glyceraldehyde 3-phosphate dehydrogenase (GAPDH; rat, dilution 1:500; Huabio; cat. no. ET1601-4). After being washed, the membranes were incubated with secondary antibody goat anti-mouse IgG (dilution, 1:1,000; Millipore; cat. no. 401211). Band intensity was quantified by Image-Pro Plus 6.0 software (Media Cybernetic).
Statistical analysis. All experiments were performed in triplicate. All data are expressed as mean \pm SD. One-way analysis of variance (ANOVA) was used for multiple-group experiments, followed by the Tukey's test, using SPSS 22.0 software (IBM Corp., Armonk, NY, USA). Significance was set at a probability value of $\mathrm{P}<0.05$.

\section{Results}

Theaflavin shows neuroprotective effects in rats subjected to cerebral I/R injury. To explore the neuroprotective effects of theaflavin, its effects in regards to reducing neurological deficits in rats of the MCAO model were investigated. As shown in Fig. 1A, the MCAO model rats had a significantly higher infarct volume than those in the sham group $(\mathrm{P}<0.01)$, indicating that the MCAO model was successfully constructed. The mean infarct volume of rats treated with theaflavin was significantly smaller than that of the untreated $(0 \mathrm{mg} / \mathrm{kg})$ MCAO model rats and also showed a significant declining trend as the dose of theaflavin increased (Fig. 1A). Furthermore, the results in Fig. 1B revealed that theaflavin treatment significantly reduced brain water content with in a dose-dependent manner. Brain water content in the MCAO model rats that received theaflavin at $50 \mathrm{mg} / \mathrm{kg}$ was significantly lower than that in rats that received theaflavin at $10 \mathrm{mg} / \mathrm{kg}(\mathrm{P}<0.01)$. We further assessed the effects of theaflavin on the neurological deficit score, learning and memory ability. As shown in Fig. 1C and D, theaflavin treatment markedly reduced the neurological deficit score and escape latency. However, severe neurological deficits and escape latency were significantly enhanced in the MCAO model $(0 \mathrm{mg} / \mathrm{kg})$ relative to the sham group $(\mathrm{P}<0.01)$. The TUNEL results in Fig. $1 \mathrm{E}$ and $\mathrm{F}$ demonstrated that the number of TUNEL-positive cells was significantly increased in the MCAO model rats $(0 \mathrm{mg} / \mathrm{kg})$ compared with rats in the sham group $(\mathrm{P}<0.01)$. In contrast to rats in the MCAO group $(0 \mathrm{mg} / \mathrm{kg})$, rats treated with theaflavin 7 days after MCAO showed a significantly reduced number of TUNEL-positive cells in a dose-dependent manner $(\mathrm{P}<0.01)$ (Fig. 1E and $\mathrm{F}$ ). The above data indicate that theaflavin has neuroprotective effects against cerebral I/R injury.

Theaflavin promotes the proliferation of NSCs and inhibits apoptosis. To elucidate the repair function of theaflavin in neural function and cognitive impairment, we investigated its effects on the proliferation and apoptosis of NSCs. The NSCs from the hippocampus were isolated as shown in Fig. 2A, and results revealed that theaflavin treatment ( 2 and $10 \mu \mathrm{M})$ significantly increased the neurosphere diameters of the NSCs subjected to OGD/R $(\mathrm{P}<0.01)$ (Fig. $2 \mathrm{~B}$ and $\mathrm{C})$. The CCK-8 results (Fig. 2D) also demonstrated that theaflavin treatment ( 2 and $10 \mu \mathrm{M})$ significantly enhanced the cell viability of NSCs subjected to OGD/R in a time-dependent manner. Moreover, with an increasing dose of theaflavin, NSC viability was significantly increased $(\mathrm{P}<0.01)$ (Fig. 2D). After OGD/R, the apoptotic rate in NSCs was significantly higher than that in the control group $(\mathrm{P}<0.01)$. Notably, theaflavin treatment $(2$ and $10 \mu \mathrm{M})$ significantly reduced the apoptotic rate in NSCs subjected to OGD/R $(\mathrm{P}<0.01)$ (Fig. 2E and F). These data indicate that theaflavin treatment promotes NSC proliferation and inhibits NSC apoptosis, 
A

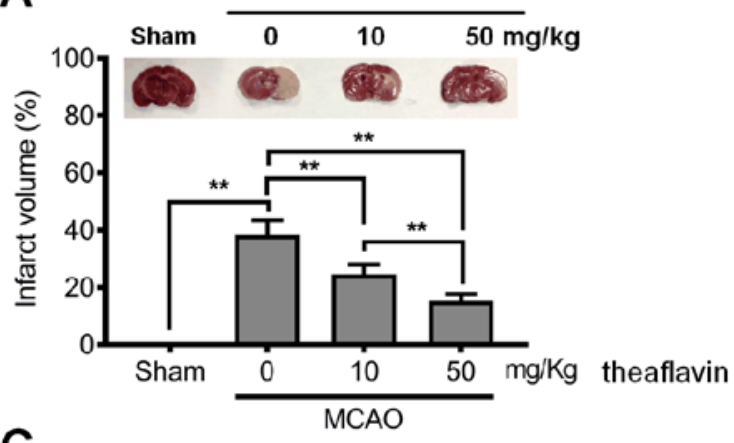

C

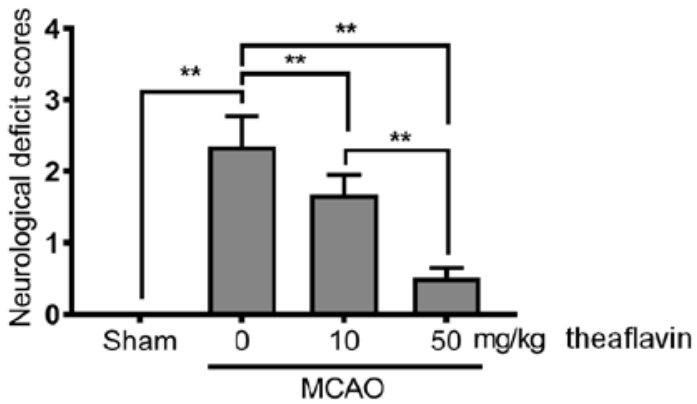

E

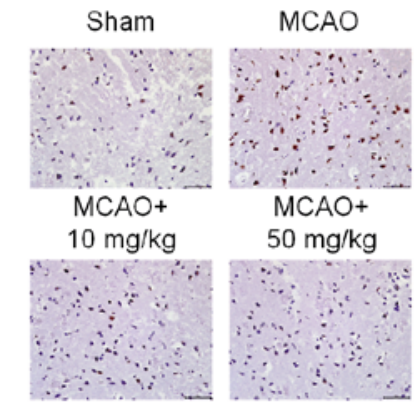

B

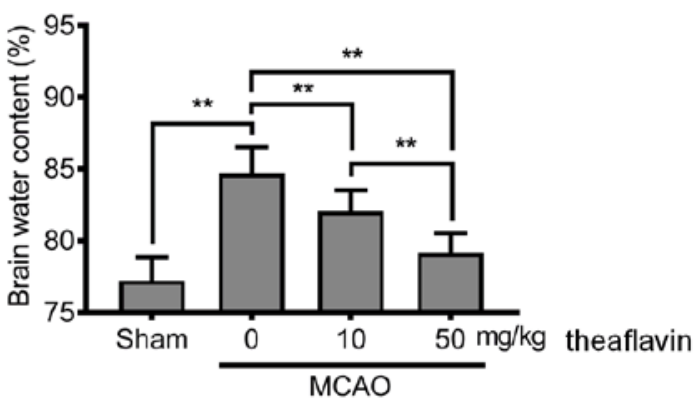

D

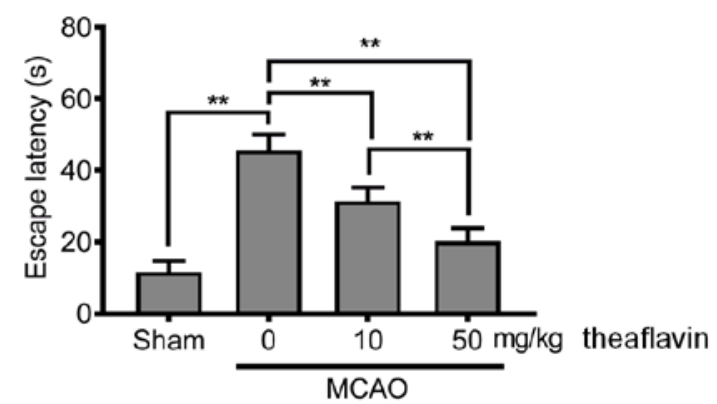

F

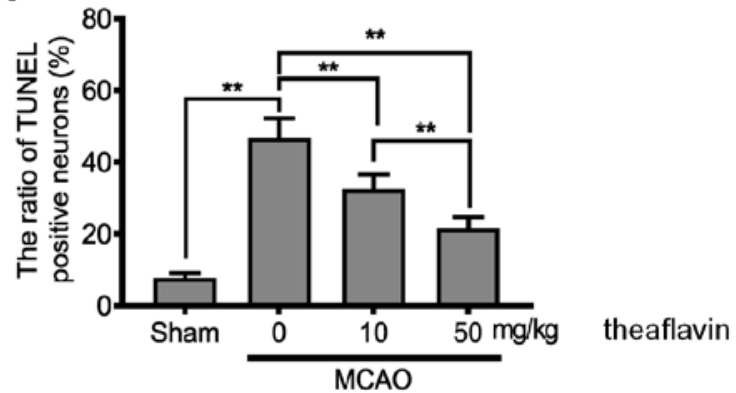

Figure 1. Theaflavin attenuates cerebral I/R injury in an MCAO rat model. (A) Effects of theaflavin $(10 \mathrm{and} 50 \mathrm{mg} / \mathrm{kg}) \mathrm{on}$ ischemic hemisphere volume in the rat brain and representative TTC-stained coronal brain sections of rats. (B) Effects of theaflavin (10 and $50 \mathrm{mg} / \mathrm{kg}$ ) on brain water content $24 \mathrm{~h}$ after cerebral $\mathrm{I} / \mathrm{R}$ injury in rats. (C) Effects of theaflavin (10 and $50 \mathrm{mg} / \mathrm{kg}$ ) on neurological deficit $24 \mathrm{~h}$ after cerebral I/R in rats. (D) Effects of theaflavin (10 and $50 \mathrm{mg} / \mathrm{kg}$ ) on escape latency $24 \mathrm{~h}$ after cerebral I/R in rats. (E) TUNEL staining of the ischemic penumbra (magnification, $\mathrm{x} 200$ ). (F) Statistical analysis of apoptotic neurons in the ischemic penumbra. All assays were performed in triplicate. Data are expressed as mean $\pm \mathrm{SD}$. ${ }^{* *} \mathrm{P}<0.01$. I/R, ischemia-reperfusion; MCAO, middle cerebral artery occlusion; TTC, 2,3,5-triphenyltetrazolium chloride; TUNEL, terminal deoxynucleotidyl transferase dUTP nick end labeling.

which would favor the recovery of neural function and repair of cognitive impairment.

Theaflavin alleviates oxidative stress in MCAO model rats and NSCs subjected to $O G D / R$. To investigate the underlying mechanism of theaflavin in exerting neuroprotective effects, we detected the antioxidative effects of theaflavin. As illustrated in Table I, the production of ROS and MDA was evidently increased in the MCAO model rats. Following theaflavin treatment, the elevated levels of ROS and MDA in the MCAO model rats were significantly attenuated in a dose-dependent manner (Table I), suggesting that theaflavin is capable of suppressing ROS generation and lipid peroxidation. We then investigated the effects of theaflavin on antioxidant enzymes in MCAO model rats. The results in Table I show that the activities of SOD and glutathione (GSH-Px) in the brain were markedly suppressed. Seven days after theaflavin administration, the activities of SOD and GSH-Px were significantly elevated. The activities of antioxidants in the MCAO model rats receiving a theaflavin dose of $50 \mathrm{mg} / \mathrm{kg}$ were significantly higher than those receiving a dose of $10 \mathrm{mg} / \mathrm{kg}$ (Table I). We further explored the underlying mechanism of theaflavin in alleviating oxidative stress in vitro. We found that the production of ROS and MDA was also markedly increased in the OGD/R cellular model (Table II), and the activities of SOD and GSH-Px were significantly suppressed in the OGD/R cellular model (Table II). The elevated ROS and MDA levels and the reduced SOD and GSH-Px activities were significantly attenuated in the OGD/R cellular model after the administration of theaflavin. Taken together, these results suggest that theaflavin alleviates oxidative stress in both MCAO ischemic brains and NSCs subjected to OGD/R.

Theaflavin increases the expression of Nrf2 by downregulating miRNA-128-3p. To further delineate the molecular mechanism by which theaflavin alleviates oxidative stress, we detected the expression of $\mathrm{Nrf} 2$ in vivo and in vitro. As evident in Fig. 3A-D, the expression of Nrf2 was markedly inhibited 
A
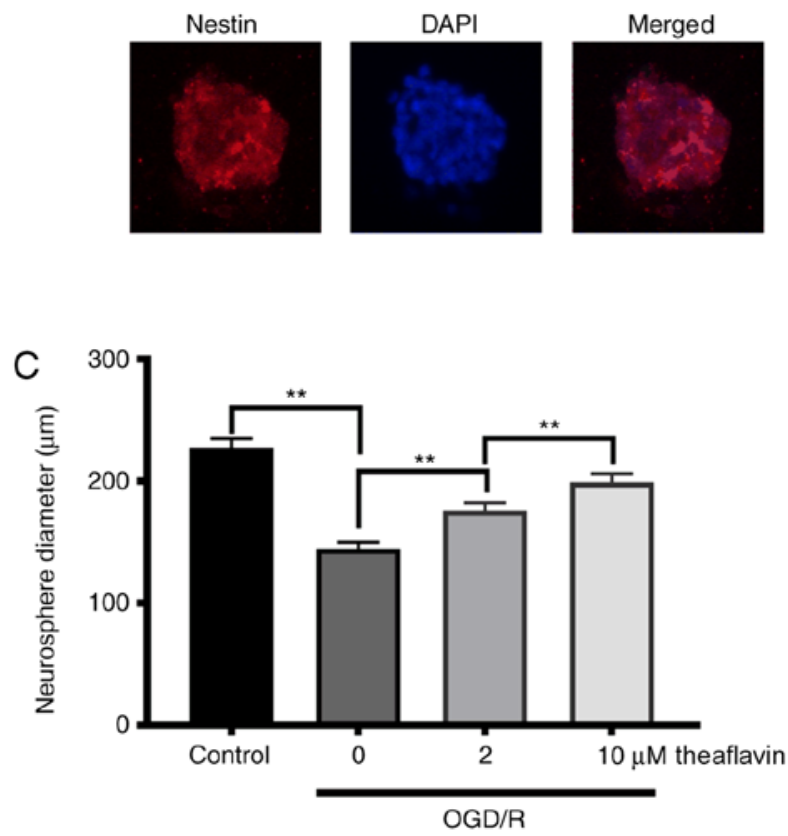

$\mathrm{E}$
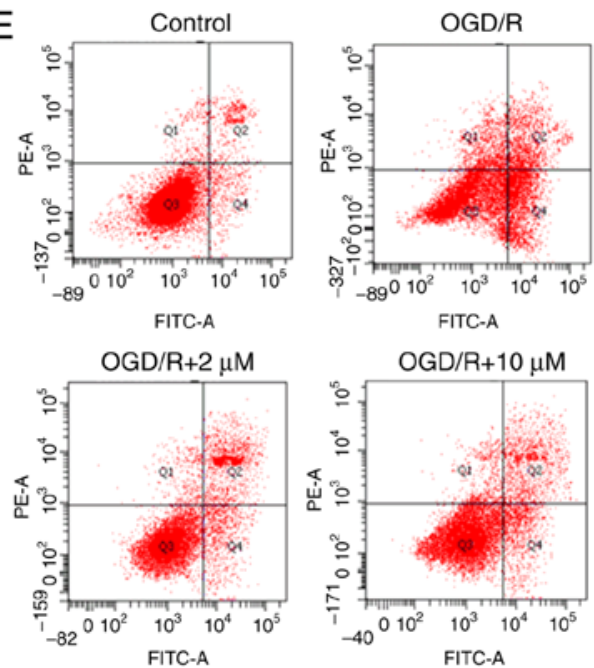

B
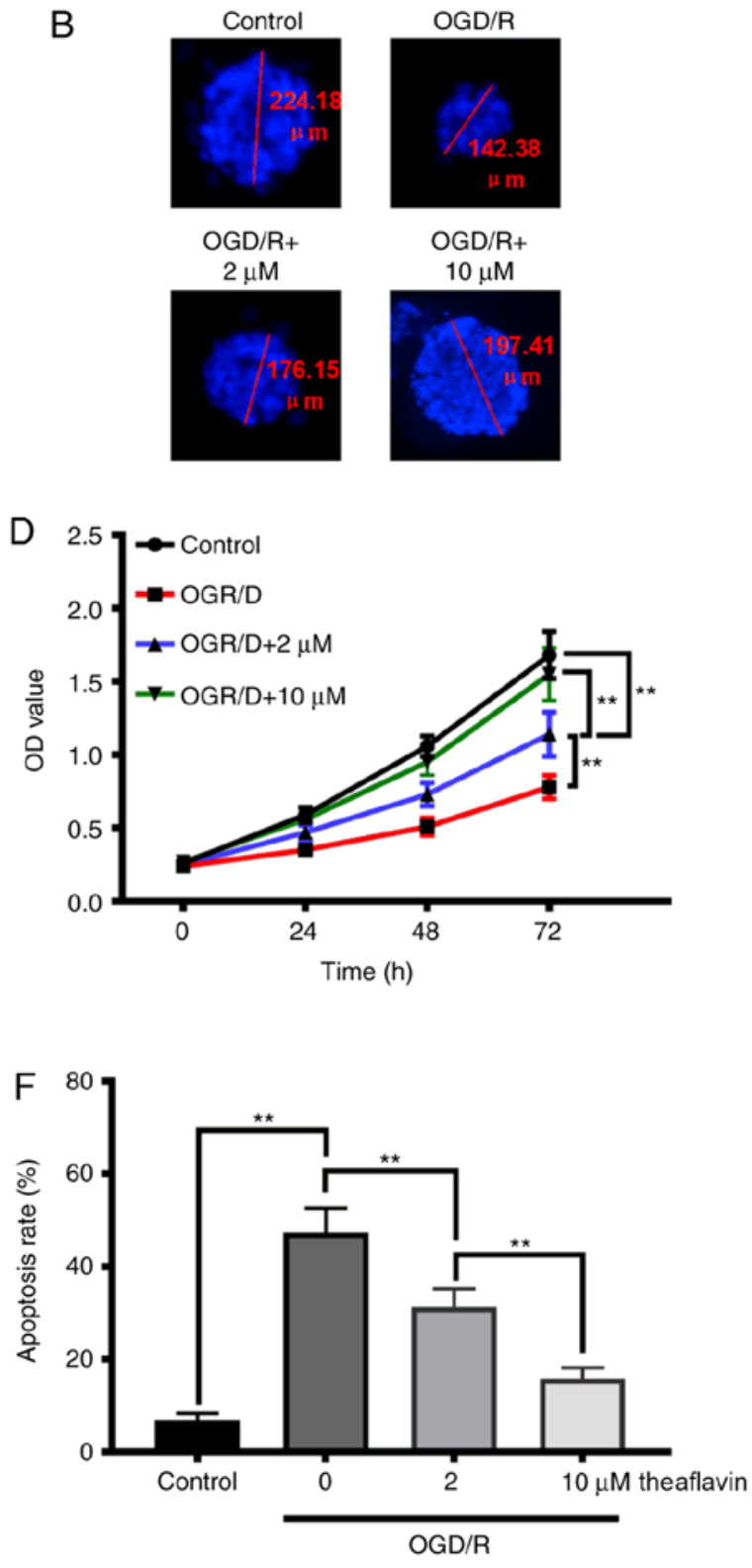

Figure 2. Effect of theaflavin on NSC viability and apoptosis. (A) Morphology of NSCs isolated from the cortex. (B) Representative neurosphere images in NSCs subjected to OGD/R with or without theaflavin treatment $(0,2$ and $10 \mu \mathrm{M})$. (C) Quantitative analysis of neurosphere diameters by ImageJ software. (D) Cell viability assay of NSCs subjected to OGD/R with or without theaflavin treatment $(0,2$ and $10 \mu \mathrm{M})$ by CCK-8 assay. (E) Apoptosis assay of NSCs subjected to OGD/R with or without theaflavin treatment $(0,2$ and $10 \mu \mathrm{M})$ by flow cytometry. (F) Quantitative analysis of the apoptotic rate. All assays were performed in triplicate. Data are expressed as mean $\pm \mathrm{SD}$. ${ }^{* *} \mathrm{P}<0.01$. NSCs, neural stem cells; OGD/R, oxygen-glucose deprivation and reoxygenation.

in MCAO model rats and NSCs subjected to OGD/R $(\mathrm{P}<0.01)$. Seven days after theaflavin administration, the expression of Nrf2 was significantly enhanced $(\mathrm{P}<0.01)$. Particularly, theaflavin at $50 \mathrm{mg} / \mathrm{kg}$ had a stronger ability to increase $\mathrm{Nrf} 2$ levels than that at $10 \mathrm{mg} / \mathrm{kg}$. Moreover, the miRNAs that target Nrf2 were investigated using bioinformatics methods. The miRNA-128-3p was predicted to regulate Nrf2 expression using the TargetScan tool (Fig. 3E). The results in Fig. 3F demonstrated that the miRNA-128-3p level was significantly upregulated in the MCAO model rats $(0 \mathrm{mg} / \mathrm{kg}$ theaflavin) compared to the Sham group $(\mathrm{P}<0.01)$. After transfection of NSCs with the miRNA mimics or miRNA inhibitor, the change in relative expression levels of miRNA-128-3p were significantly increased or decreased, respectively (Fig. 3G).
A significant increase in miRNA-128-3p levels was also found in the NSCs subjected to OGD/R $(\mathrm{P}<0.01)$ (Fig. 4H). Notably, we found that theaflavin reduced the level of miRNA-128-3p levels significantly in both the MCAO and OGD/R NSC models in a dose-dependent manner $(\mathrm{P}<0.01)$ (Fig. 3F and H). The results presented in Fig. 3H also indicated that miRNA mimics $+(10 \mu \mathrm{M})$ theaflavin treatment increased the level of miRNA-128-3p, in contrast to our observations in NSCs subjected to OGD/R, indicating that theaflavin induced the downregulation of miRNA-128-3p. To confirm whether miRNA-128-3p inhibited Nrf2 expression, a dual-luciferase reporter assay was employed using the wild-type (WT) site or the mutant (MUT) site. A considerable decline in luciferase activity was evident with 
Table I. Theaflavin inhibits oxidative stress in the rat brain.

\begin{tabular}{llccc}
\hline Group & ROS (F/F0) & MDA (mmol/mg) & SOD (U/mg) & GSH-Px (U/mg) \\
\hline Sham & $1.38 \pm 0.14$ & $6.78 \pm 0.86$ & $125.73 \pm 14.38$ & $17.71 \pm 3.35$ \\
MCAO + theaflavin (0 mg/kg) & $2.34 \pm 0.27^{\mathrm{a}}$ & $15.66 \pm 1.38^{\mathrm{a}}$ & $58.44 \pm 7.83^{\mathrm{a}}$ & $8.63 \pm 1.02^{\mathrm{a}}$ \\
MCAO + theaflavin $(10 \mathrm{mg} / \mathrm{kg})$ & $1.97 \pm 0.21^{\mathrm{a}, \mathrm{b}}$ & $13.05 \pm 1.15^{\mathrm{a}, \mathrm{b}}$ & $87.59 \pm 9.57^{\mathrm{a}, \mathrm{b}}$ & $12.58 \pm 1.69^{\mathrm{a}, \mathrm{b}}$ \\
MCAO + theaflavin $(50 \mathrm{mg} / \mathrm{kg})$ & $1.58 \pm 0.17^{\mathrm{a}-\mathrm{c}}$ & $9.04 \pm 1.02^{\mathrm{a}-\mathrm{c}}$ & $103.26 \pm 13.21^{\mathrm{a}-\mathrm{c}}$ & $14.36 \pm 2.07^{\mathrm{a}-\mathrm{c}}$
\end{tabular}

${ }^{\mathrm{a}} \mathrm{P}<0.01$ vs. the sham group; ${ }^{\mathrm{b}} \mathrm{P}<0.01$ vs. the $\mathrm{MCAO}+$ theaflavin $(0 \mathrm{mg} / \mathrm{kg})$ group; ${ }^{\mathrm{C}} \mathrm{P}<0.01 \mathrm{vs}$. the $\mathrm{MCAO}+$ theaflavin $(10 \mathrm{mg} / \mathrm{kg}) \mathrm{group}$. $\mathrm{MCAO}$, middle cerebral artery occlusion; ROS, reactive oxygen species; MDA, malondialdehyde; GSH-Px, glutathione peroxidase; SOD, superoxide dismutase.

Table II. Theaflavin inhibits oxidative stress in NSCs.

\begin{tabular}{|c|c|c|c|c|}
\hline Group & ROS (F/F0) & $\mathrm{MDA}(\mathrm{mmol} / \mathrm{mg})$ & $\mathrm{SOD}(\mathrm{U} / \mathrm{mg})$ & GSH-Px (U/mg) \\
\hline Control & $1.21 \pm 0.13$ & $1.45 \pm 0.26$ & $16.49 \pm 2.36$ & $1.52 \pm 0.18$ \\
\hline OGD/R + theaflavin $(0 \mathrm{mg} / \mathrm{kg})$ & $2.69 \pm 0.30^{\mathrm{a}}$ & $2.93 \pm 0.34^{\mathrm{a}}$ & $6.38 \pm 1.05^{\mathrm{a}}$ & $0.63 \pm 0.09^{\mathrm{a}}$ \\
\hline OGD/R + theaflavin $(2 \mathrm{mg} / \mathrm{kg})$ & $2.12 \pm 0.24^{\mathrm{a}, \mathrm{b}}$ & $2.16 \pm 0.18^{\mathrm{a}, \mathrm{b}}$ & $8.97 \pm 1.25^{\mathrm{a}, \mathrm{b}}$ & $0.91 \pm 0.11^{\mathrm{a}, \mathrm{b}}$ \\
\hline OGD/R + theaflavin $(10 \mathrm{mg} / \mathrm{kg})$ & $1.64 \pm 0.19^{\mathrm{a}-\mathrm{c}}$ & $1.74 \pm 0.21^{\mathrm{a}-\mathrm{c}}$ & $13.26 \pm 1.68^{\mathrm{a}-\mathrm{c}}$ & $1.34 \pm 0.15^{\mathrm{a}-\mathrm{c}}$ \\
\hline
\end{tabular}

${ }^{a} \mathrm{P}<0.01$ vs. the control group; ${ }^{\mathrm{P}} \mathrm{P}<0.01 \mathrm{vs}$. the $\mathrm{OGD} / \mathrm{R}+$ theaflavin $(0 \mathrm{mg} / \mathrm{kg})$ group; ${ }^{\mathrm{C}} \mathrm{P}<0.01 \mathrm{vs}$. the $\mathrm{OGD} / \mathrm{R}+$ theaflavin $(2 \mathrm{mg} / \mathrm{kg}) \mathrm{group}$. NSCs, neural stem cells; OGD/R, oxygen-glucose deprivation and reoxygenation; ROS, reactive oxygen species; MDA, malondialdehyde; GSH-Px, glutathione peroxidase; SOD, superoxide dismutase.

co-transfection of the miRNA-128-3p mimic and reporter plasmid at the Nrf2 WT site (Fig. 3I) $(\mathrm{P}<0.01)$. However, a slight reduction in luciferase activity was observed after co-transfection with the miRNA-128-3p mimic and reporter plasmid at the Nrf2 MUT site (Fig. 3I). To further demonstrate the effect of miRNA-128-3p on Nrf2, the levels of $N r f 2$ mRNA and protein were measured using qRT-PCR and western blot analysis. As shown in Fig. 3J and K, ectopic expression of miRNA-128-3p induced a significant decline in the levels of $N r f 2 \mathrm{mRNA}$ and protein. These results indicate that theaflavin downregulates miRNA-128-3p to increase Nrf2 levels to alleviate oxidative stress.

Inhibition of NSC proliferation and enhancement of oxidative stress by miRNA-128-3p. To further confirm the regulatory function of miRNA-128-3p, we examined its effects on NSC proliferation. Furthermore, we also upregulated the expression of $\mathrm{Nrf} 2$ (Fig. 4A). The results presented in Fig. 4B-D demonstrated that the application of the miRNA-128-3p inhibitor significantly increased the neurosphere diameter and cell viability of the NSCs subjected to OGD/R $(\mathrm{P}<0.01)$. Notably, both the ectopic expression of miRNA-128-3p and Nrf2 slightly increased the neurosphere diameter and cell viability of NSCs subjected to OGD/R $(\mathrm{P}<0.01)$. Furthermore, it was found that upregulation of Nrf2 (OE-Nrf2) was capable of increasing the neurosphere diameter and cell viability, an effect that was abolished after treatment with miRNA-128-3p mimics (Fig. 4B-D). As shown in Fig. 4E and F, the elevated apoptotic rate in NSCs was significantly attenuated by the miRNA-128-3p inhibitor $(\mathrm{P}<0.01)$. Whereas the apoptotic rate in NSCs receiving both miRNA-128-3p mimics and overexpression of Nrf2 was slightly reduced compared with that in NSCs subjected to OGD/R ( $\mathrm{P}>0.05)$. In addition, upregulation of Nrf2 showed a stronger ability to reduce the rate of apoptosis, an effect which was abolished after treatment with miRNA-128-3p mimics $(\mathrm{P}<0.01)$. These findings indicate that downregulation of miRNA-128-3p promoted NSC proliferation by upregulating Nrf2 expression.

We investigated the effect of miRNA-128-3p on oxidative stress in NSCs subjected to OGD/R. The levels of ROS and MDA were significantly reduced after treatment with miRNA-128-3p inhibitors $(\mathrm{P}<0.01)$ (Fig. 4G and $\mathrm{H}$ ), and were slightly reduced after treatment with miRNA-128-3p mimics and theaflavin, the elevated ROS and MDA levels were slightly attenuated. As expected, upregulation of Nrf2 significantly inhibited the production of ROS and MDA, an effect which was abolished after treatment with miRNA-128-3p mimics (Fig. 4G and $\mathrm{H}$ ). These data suggest that reducing miRNA-128-3p levels is beneficial for the inhibition of ROS generation and lipid peroxidation. Further studies showed that the miRNA-128-3p inhibitor and elevated Nrf2 levels significantly upregulated the levels of antioxidant enzymes, including GSH-Px and SOD (Fig. 4I and J). Particularly, treatment with miRNA-128-3p mimics and Nrf2 overexpression were unable to increase the levels of the antioxidant enzymes ( $\mathrm{P}>0.05)$ (Fig. 4I and $\mathrm{J}$ ). Collectively, these results indicate that reduced miRNA-128-3p levels are beneficial to the restoration of an impaired antioxidant defense system. 
A

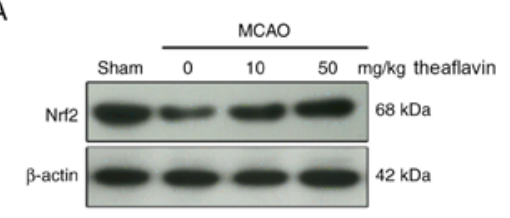

C

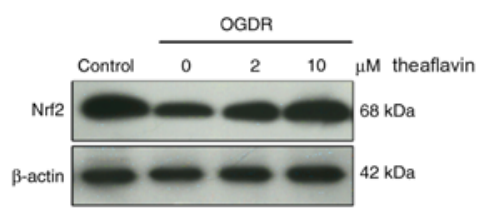

E

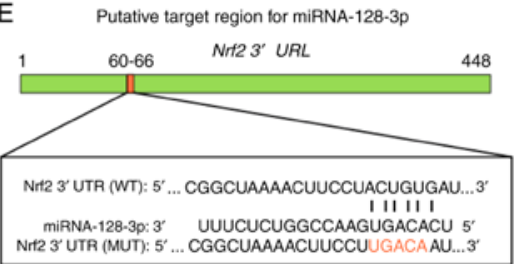

G

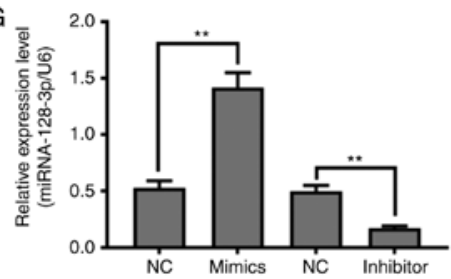

।

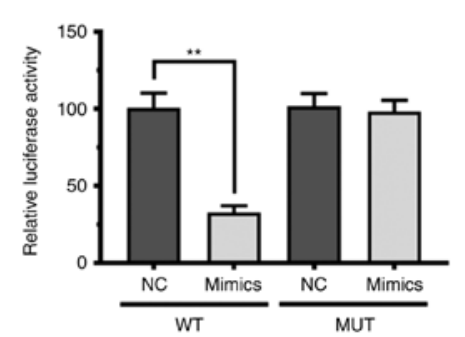

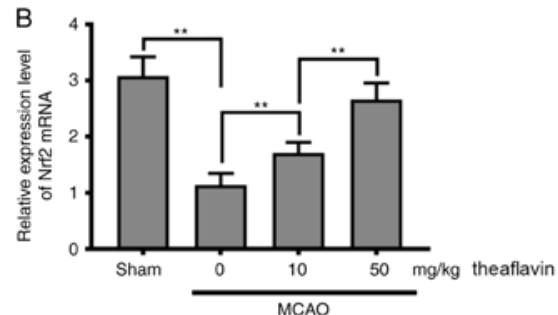
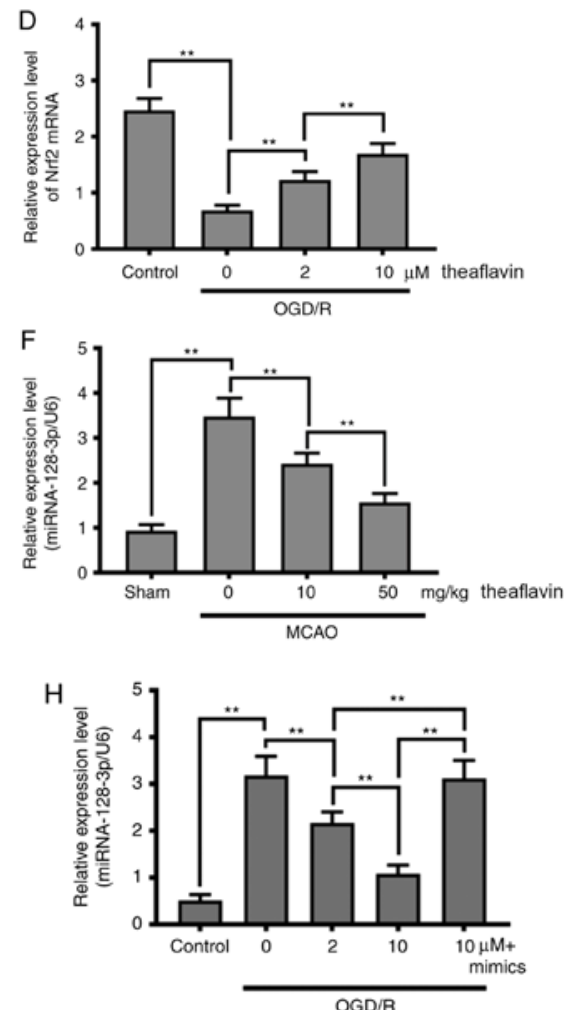

J

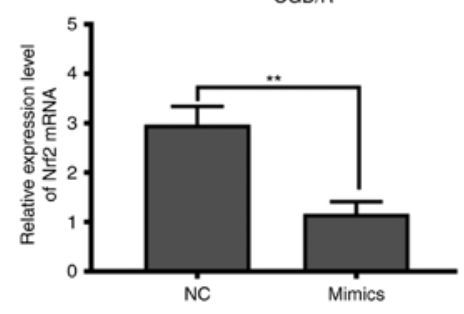

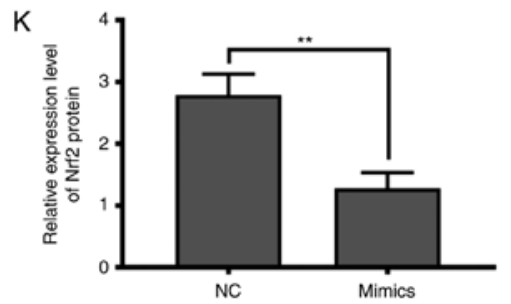

Figure 3. Direct inhibition of Nrf2 expression by miRNA-128-3p via targeting of the 3' UTR in NSCs. (A) Western blot analysis shows the Nrf2 levels $24 \mathrm{~h}$ after cerebral $\mathrm{I} / \mathrm{R}$ in the MCAO model with or without theaflavin treatment $(0,10$ and $50 \mathrm{mg} / \mathrm{kg})$. $\beta$-actin was used as the internal control for normalization. (B) RT-qPCR analysis shows Nrf2 mRNA levels $24 \mathrm{~h}$ after cerebral I/R in the MCAO model with or without theaflavin treatment $(0,10 \mathrm{and} 50 \mathrm{mg} / \mathrm{kg}) . \mathrm{GAPDH}$ mRNA was used as the internal control for normalization. (C) Western blot analysis shows Nrf2 levels in NSCs subjected to OGD/R with or without theaflavin treatment $(0,2$ and $10 \mu \mathrm{M})$. $\beta$-actin was used as the internal control for normalization. (D) RT-qPCR analysis shows Nrf2 mRNA levels in NSCs subjected to OGD/R with or without theaflavin treatment $(0,2$ and $10 \mu \mathrm{M})$. GAPDH mRNA was used as the internal control for normalization. (E) Sequence alignment between miRNA-128-3p and the 3'UTR of Nrf2 mRNA. (F) RT-qPCR analysis shows miRNA-128-3p levels $24 \mathrm{~h}$ after cerebral I/R in the MCAO model with or without theaflavin treatment $(0,10$ and $50 \mathrm{mg} / \mathrm{kg})$. U6 was used as the internal control for normalization. (G) RT-qPCR analysis shows miRNA-128-3p levels after transfection of miRNA mimics or miRNA inhibitor. (H) RT-qPCR analysis shows miRNA-128-3p levels in NSCs subjected to OGD/R with or without theaflavin treatment $(0,2$ and $10 \mu \mathrm{M})$ or with $10 \mu \mathrm{M}$ theaflavin and miRNA mimic. U6 was used as the internal control for normalization. (I) The luciferase reporter gene assay shows the effect of miRNA-128-3p on luciferase activity. (J) RT-qPCR analysis shows Nrf2 mRNA levels in NSCs subjected to OGD/R after transfection of mimics (K) Western blot analysis shows the Nrf2 levels in NSCs subjected to OGD/R after transfection of mimics. All assays were performed in triplicate. Data are expressed as mean $\pm \mathrm{SD}$. ${ }^{* *} \mathrm{P}<0.01$. Nrf2, nuclear factor (erythroid-derived 2)-related factor 2; UTR, untranslated region; NSCs, neural stem cells; I/R, ischemia-reperfusion; MCAO, middle cerebral artery occlusion; OGD/R, oxygen-glucose deprivation and reoxygenation. 
A
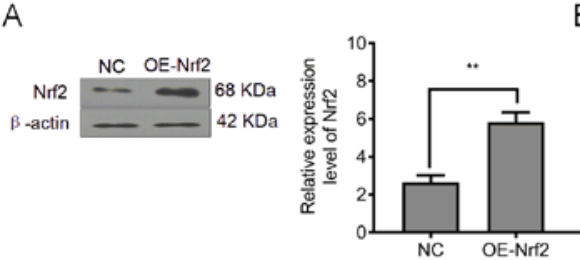

C

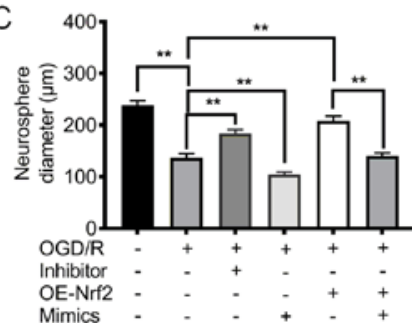

E
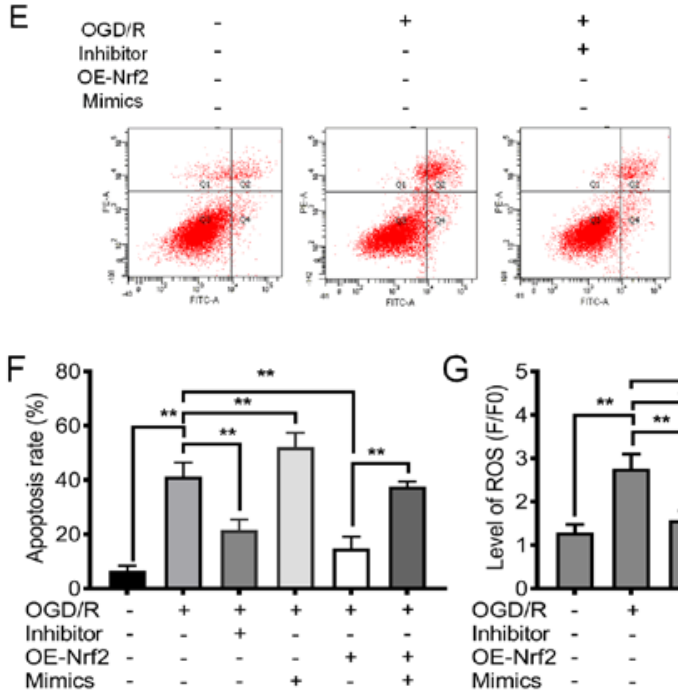

G

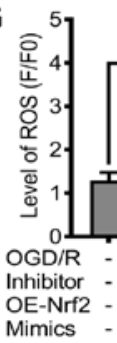

I

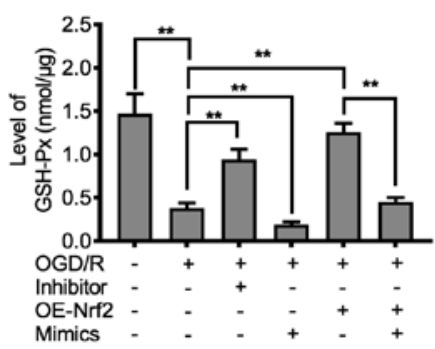

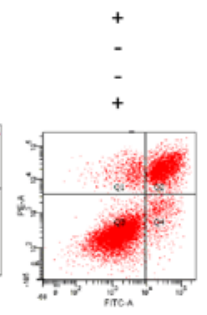
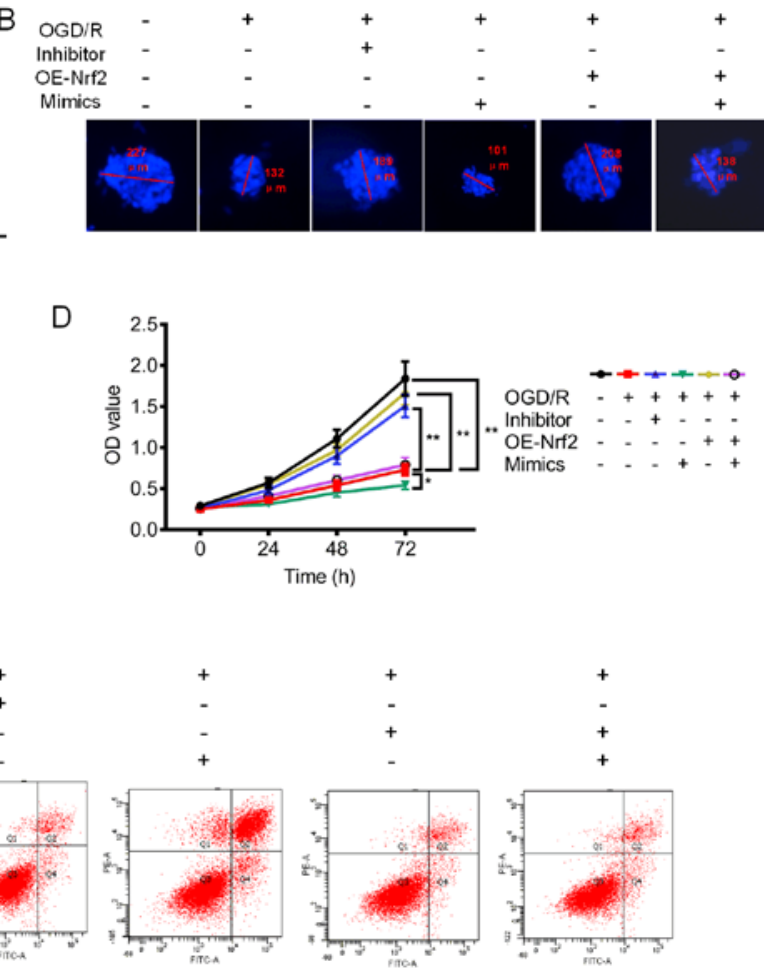

D
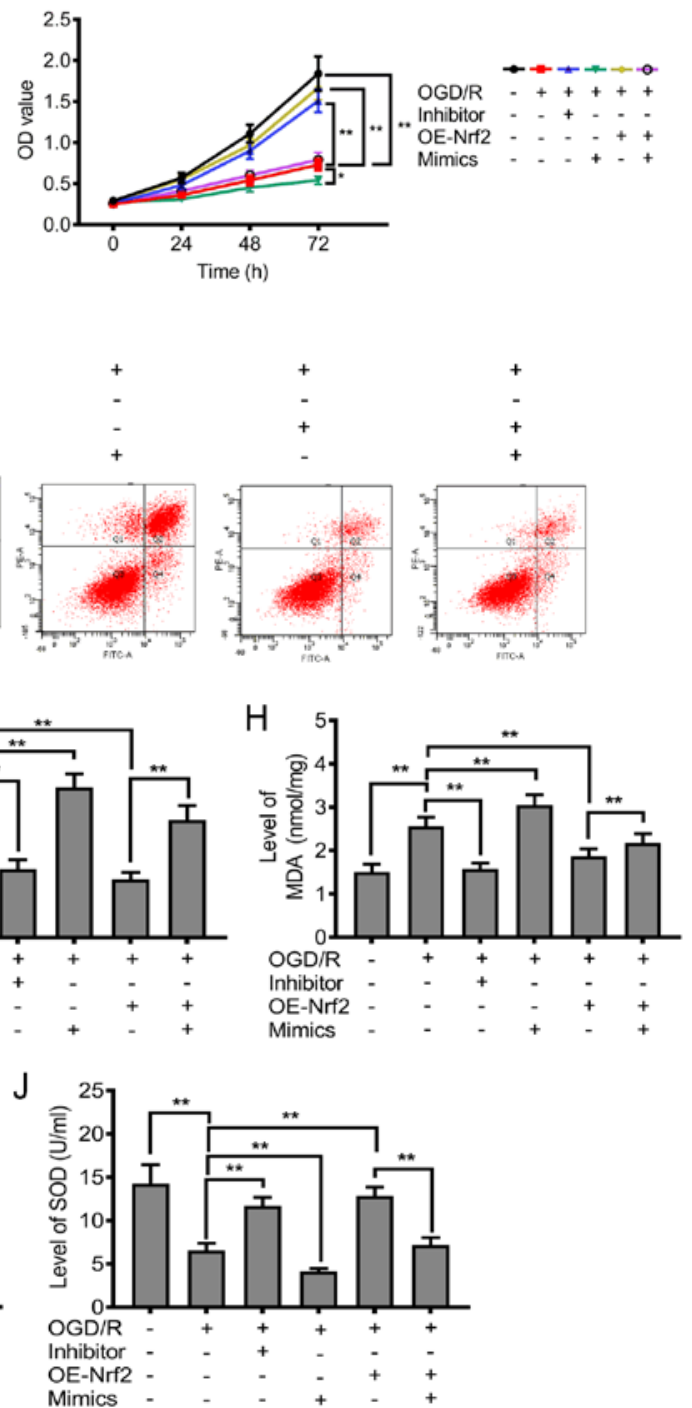

Figure 4. Downregulation of miRNA-128-3p and upregulation of Nrf2 promote cell proliferation and inhibit apoptosis and oxidative stress. (A) Relative expression level of Nrf2 after upregulation of Nrf2 (OE-Nrf2). (B) Representative neurosphere images in NSCs subjected to OGD/R after downregulation or upregulation of miRNA-128-3p (with inhibitor and mimics, respectively), or upregulation of Nrf2 (OE-Nrf2). (C) Quantitative analysis of neurosphere diameter by Image J software. (D) Cell Counting Kit-8 (CCK-8) assay shows the cell viability of NSCs subjected to OGD/R after downregulation or upregulation of miRNA-128-3p (with inhibitor and mimics, respectively), or upregulation of Nrf2 (OE-Nrf2). (E) Flow cytometry shows the apoptosis of NSCs subjected to OGD/R after downregulation of miRNA-128-3p (Inhibitor) or upregulation of Nrf2 (OE-Nrf2). (F) Quantitative analysis of the apoptotic rate. (G) ROS levels in NSCs subjected to OGD/R after after downregulation or upregulation of miRNA-128-3p (with inhibitor and mimics, respectively), or upregulation of Nrf2 (OE-Nrf2). (H) MDA content in NSCs subjected to OGD/R after downregulation of miRNA-128-3p (Inhibitor) or upregulation of Nrf2 (OE-Nrf2). (I) GSH-Px levels in NSCs subjected to OGD/R after downregulation or upregulation of miRNA-128-3p (with inhibitor and mimics, respectively), or upregulation of Nrf2 (OE-Nrf2). (J) SOD activity in NSCs subjected to OGD/R after downregulation of miRNA-128-3p (Inhibitor) or upregulation of Nrf2 (OE-Nrf2). All assays were performed in triplicate. Data are expressed as the mean $\pm \mathrm{SD} .{ }^{*} \mathrm{P}<0.05,{ }^{* *} \mathrm{P}<0.01$. Nrf2, nuclear factor (erythroid-derived 2)-related factor 2; NSCs, neural stem cells; OGD/R, oxygen-glucose deprivation and reoxygenation; MDA, malondialdehyde; GSH-Px, glutathione peroxidase; SOD, superoxide dismutase.

\section{Discussion}

Nowadays, ischemic stroke presents a considerable social and family burden. Although the reperfusion of ischemic cerebral tissue is the most common way to rescue ischemic stroke, CI/RI can further lead to brain damage and even death $(24,25)$. Therefore, new strategies must be developed to prevent $\mathrm{CI} / \mathrm{RI}$. In recent years, plant extracts have garnered increasing attention from academia and the public. Previously, studies have shown that theaflavin is a promising active compound from black tea that can regulate inflammation and oxidative stress $(17,26)$. However, the biological function and 
the molecular mechanism of theaflavin in ameliorating ischemic damage has not yet been fully elucidated. In the present study, we explored the biological function of theaflavin in ameliorating CI/RI and investigated its potential molecular mechanism. We found that theaflavin exerts neuroprotective effects by reducing infarct volume and improving learning and memory in rats subjected to CI/RI. Furthermore, we provided convincing evidence that theaflavin treatment can reduce apoptosis in NSCs and promote NSC proliferation. These findings were supported by the results of the MCAO injury rat model, as well as those of the cultured NSCs subjected to OGD/R. Importantly, we proved that theaflavin could be a Nrf2 promoter by downregulating miRNA-128-3p, which effectively inhibited oxidative stress in rats subjected to CI/RI and NSCs subjected to OGD/R. Our results collectively indicate that theaflavin could be a promising natural compound for the attenuation of CI/RI. To our knowledge, we found a new molecular mechanism for the neuroprotective function of theaflavin downregulating miRNA-128-3p to inhibit oxidative stress to further promote repair following ischemic stroke.

Most of the biological functions of tea polyphenols, including theaflavins and catechins, have been ascribed to their anti-oxidative, anti-tumor, anti-bacterial, anti-inflammatory, anti-viral, and cardiovascular protective activities (27-29). For example, Zhang et al found that theaflavin showed neuroprotective effects against oxidative stress in PC12 cells, which was derived from the suppression of oxidant enzyme activity (16). In the present study, theaflavin treatment commenced during the development of CI/RI in rats. Theaflavin was evidently capable of reducing infarct volume and ameliorating symptoms related to CI/RI. Importantly, these inhibitory effects of theaflavin were observed in a dose-dependent manner. Our results indicate that theaflavin has a neuroprotective function in CI/RI. To further investigate the role of theaflavin in its neuroprotective effects in CI/RI, TUNEL staining was used in the present study. Theaflavin decreased the rate of apoptosis in the brain tissue of rats subjected to CI/RI. Recently, several research studies have shown that neurogenesis is insufficient for functional recovery in ischemic stroke disability $(30,31)$. Therefore, targeting endogenous NSCs to promote adult neurogenesis is a feasible strategy for the recovery of nervous function and repair of cognitive impairment in ischemic stroke. We isolated NSCs from the hippocampus, which is responsible for long-term memory storage conversion and orientation functions. Following theaflavin treatment, the cell viability of NSCs subjected to OGD/R was significantly increased and the apoptosis rate showed a significant decline. These results further indicate that theaflavin has a neuroprotective function.

Oxidative stress is a critically important pathological process in CI/RI that contributes to neurocyte injury (32). Neural tissue is vulnerable to oxidative attack, owing to its high consumption of oxygen, relatively low antioxidant capacity, and high polyunsaturated fatty acid content (33). Moreover, oxidative injury of mitochondrial DNA would lead to impaired energy regulation, which is critical in high-energy-requiring neurons $(8,34)$. Current research has shown that plant extracts exert strong neuroprotective effects by inhibiting oxidative stress. As a result of the anti-oxidative function of theaflavin, we explored its role in inhibiting oxidative stress in CI/RI. Seven days after theaflavin treatment, the activities of antioxidants in both the MCAO model rats and NSCs subjected to OGD/R were significantly elevated. In addition, the elevated ROS and MDA levels were significantly attenuated in both the MCAO model rats and NSCs subjected to OGD/R after theaflavin treatment, indicating that theaflavin also exhibits a stronger anti-oxidative capacity in CI/RI. Our findings are largely consistent with a recent report, in which theaflavins showed significant protective effects in erythrocytes against oxidative stress induced by tert-butyl hydroperoxide by increasing GSH-Px and reducing MDA (35). The alleviation of oxidative stress by theaflavin is responsible for NSC proliferation and anti-apoptosis, which aids in the recovery of nervous function and the repair of cognitive impairment. The Nrf2-dependent pathway plays a critical role in oxidative stress. Recent research reported that theaflavin could ameliorate ionizing radiation-induced hematopoietic stem cell injury via the Nrf2 pathway (36). Thus, the expression of Nrf2 in CI/RI was investigated. We found that the Nrf2 protein and Nrf 2 mRNA levels were obviously up-regulated by theaflavin in both the MCAO rat model and NSCs subjected to OGD/R. Thus, we believe that theaflavin may attenuate $\mathrm{CI} / \mathrm{RI}$ by inhibiting oxidative stress via the Nrf2-dependent pathway. These results suggest the feasibility of theaflavin as a treatment for ischemic stroke and neurological recovery.

The miRNAs are crucial regulators in spontaneous neurogenesis and sustainability of neuronal phenotype by post-transcriptional regulation. Furthermore, miRNAs, including miRNA-23a-3p, miRNA-424, and miRNA-125b reportedly reduce $\mathrm{CI} / \mathrm{RI}$ by targeting the oxidative stress pathway (37-39). Based on these functions of miRNA, Nrf2 mRNA/miRNA interaction was predicted using the miRNA target prediction tool, TargetScan, and Nrf2 mRNA/miRNA-128-3p interaction information was also predicted. Our results showed that the significant increase in miRNA-128-3p was eliminated following theaflavin treatment in both the MCAO ischemic rats and NSCs subjected to OGD/R. Interestingly, ectopic expression of miRNA-128-3p resulted in a significant decline in the levels of $N r f 2$ mRNA and protein, which further confirmed the regulatory effect of miRNA-128-3p on Nrf2. Thus, theaflavin improved the expression of Nrf2 by downregulating miRNA-128-3p. Several studies have shown that abnormal expression of miRNA-128-3p is closely related to I/RI, contextual learning, and DNA damage response $(40,41)$. For example, Chen et al found that inhibition of miR-128-3p could protect human cardiomyocytes from I/RI (42). Loss-of-function experiments in our study showed that downregulation of miRNA-128-3p promoted NSC proliferation and reduced the rate of apoptosis, which is consistent with previous reports that antagonism of miR-128-3p could remarkably improve the proliferation of lymphatic endothelial cells (43). Furthermore, miRNA-128-3p exhibited opposite regulatory effects to Nrf2 in oxidative stress. Thus, downregulation of miRNA-128-3p expression along with theaflavin treatment contribute to the attenuation of CI/RI. Chen et al demonstrated that the downregulation of miRNA-128-3p in mouse hippocampi promoted the differentiation of NSCs in the performance of the water maze task (41). However, the research of Mao et al showed the opposite conclusion, as they reported that an increase in miRNA-128-3p levels contributed to neuronal survival in ischemia-induced brain injury (44). Such inconsistency may be that due to different animal models and experimental conditionsled to a disparity in 
observations.Our findings present a comprehensive understanding of the underlying mechanism of theaflavin in ischemic stroke.

In conclusion, theaflavin could effectively attenuate $\mathrm{CI} / \mathrm{RI}$ and improve learning and memory through miRNA-128-3p-mediated anti-oxidative actions in rats subjected to ischemia-induced brain injury and NSCs subjected to OGD/R. Further studies can focus on the regulatory mechanism of theaflavin on miRNA-128-3p expression, high-energy-requiring neurons and the effect of theaflavin on NSC differentiation. In all, theaflavin holds the potential to be a new drug candidate for ischemic stroke treatment.

\section{Acknowledgements}

Not applicable.

\section{Funding}

The present study was supported by a grant (grant no. 201640129) from Shanghai Municipal Planning Commission of science and Research Fund grant (grant no. 2018YFA0107900) from National Key R\&D Program of China; grant (grant no. 81200936) from the National Nature Science Foundation, and grant (grant no. 134119a8500) from Shanghai Committee of Science and Technology.

\section{Availability of data and materials}

All data generated or analyzed during the present study are included in this published article.

\section{Authors' contributions}

RL and JZ concieved of and designed the study. HW provided administrative support. XL and ZY collected and assembled the data. LF analysed and interpreted the data. RL wrote the manuscript. All authors read and approved the final manuscript.

\section{Ethics approval and consent to participate}

Not applicable.

\section{Patient consent for publication}

Not applicable.

\section{Competing interests}

The authors declare that they have no competing interests.

\section{References}

1. Della-Morte D, Pacifici F and Rundek T: Genetic susceptibility to cerebrovascular disease. Curr Opin Lipidol 27: 187-195, 2016.

2. Hu X, De Silva TM, Chen J and Faraci FM: Cerebral vascular disease and neurovascular injury in ischemic stroke. Circ Res 120: 449-471, 2017.

3. Gerschenfeld G, Muresan IP, Blanc R, Obadia M, Abrivard M, Piotin $\mathrm{M}$ and Alamowitch S: Two paradigms for endovascular thrombectomy after intravenous thrombolysis for acute ischemic stroke. JAMA Neurol 74: 549-556, 2017.
4. Ji K, Xue L, Cheng J and Bai Y: Preconditioning of H2S inhalation protects against cerebral ischemia/reperfusion injury by induction of HSP70 through PI3K/Akt/Nrf2 pathway. Brain Res Bull 121: 68-74, 2016.

5. Xu J, Kong X, Xiu H, Dou Y, Wu Z and Sun P: Combination of curcumin and vagus nerve stimulation attenuates cerebral ischemia/reperfusion injury-induced behavioral deficits. Biomed Pharmacother 103: 614-620, 2018.

6. Chen X, Wu H, Chen H, Wang Q, Xie XJ and Shen J: Astragaloside VI promotes neural stem cell proliferation and enhances neurological function recovery in transient cerebral ischemic injury via activating EGFR/MAPK signaling cascades. Mol Neurobiol 56: 3053-3067, 2019.

7. Wu J, Chen Y, Yu S, Li L, Zhao X, Li Q, Zhao J and Zhao Y: Neuroprotective effects of sulfiredoxin-1 during cerebral ischemia/reperfusion oxidative stress injury in rats. Brain Res Bull 132: 99-108, 2017.

8. Wang M, Li YJ, Ding Y, Zhang HN, Sun T, Zhang K, Yang L, Guo YY, Liu SB, Zhao MG and Wu YM: Silibinin prevents autophagic cell death upon oxidative stress in cortical neurons and cerebral ischemia-reperfusion injury. Mol Neurobiol 53: 932-943, 2016.

9. Guo C, Wang S, Duan J, Jia N, Zhu Y, Ding Y, Guan Y, Wei G, Yin Y, Xi M and Wen A: Protocatechualdehyde protects against cerebral ischemia-reperfusion-induced oxidative injury via protein kinase $\mathrm{C} \varepsilon / \mathrm{Nrf} 2 / \mathrm{HO}-1$ pathway. Mol Neurobiol 54: 833-845, 2017.

10. Khajevand-Khazaei MR, Azimi S, Sedighnejad L, Salari S, Ghorbanpour A, Baluchnejadmojarad T, Mohseni-Moghaddam P, Khamse S and Roghani M: S-allyl cysteine protects against lipopolysaccharide-induced acute kidney injury in the C57BL/6 mouse strain: Involvement of oxidative stress and inflammation. Int Immunopharmacol 69: 19-26, 2019.

11. Chen M, Dai LH, Fei A, Pan SM and Wang HR: Isoquercetin activates the ERK1/2-Nrf2 pathway and protects against cerebral ischemia-reperfusion injury in vivo and in vitro. Exp Ther Med 13: 1353-1359, 2017.

12. Zhang W, Wei R, Zhang L, Tan Y and Qian C: Sirtuin 6 protects the brain from cerebral ischemia/reperfusion injury through NRF2 activation. Neuroscience 366: 95-104, 2017.

13. Chang C, Zhao Y, Song G and She K: Resveratrol protects hippocampal neurons against cerebral ischemia-reperfusion injury via modulating JAK/ERK/STAT signaling pathway in rats. J Neuroimmunol 315: 9-14, 2018.

14. Jiao $\mathrm{S}$, Zhu H, He P and Teng J: Betulinic acid protects against cerebral ischemia/reperfusion injury by activating the PI3K/Akt signaling pathway. Biomed Pharmacother 84: 1533-1537, 2016.

15. Feng Q, Torii Y, Uchida K, Nakamura Y, Hara Y and Osawa T: Black tea polyphenols, theaflavins, prevent cellular DNA damage by inhibiting oxidative stress and suppressing cytochrome $\mathrm{P} 450$ 1A1 in cell cultures. J Agric Food Chem 50: 213-220, 2002.

16. Zhang J, Cai S, Li J, Xiong L, Tian L, Liu J, Huang J and Liu Z: Neuroprotective effects of theaflavins against oxidative stress-induced apoptosis in PC12 cells. Neurochem Res 41: 3364-3372, 2016.

17. Cai F, Li CR, Wu JL, Chen JG, Liu C, Min Q, Yu W, Ouyang CH and Chen JH: Theaflavin ameliorates cerebral ischemia-reperfusion injury in rats through its anti-inflammatory effect and modulation of STAT-1. Mediators Inflamm 2006: 30490, 2006.

18. Liang Q, Luo Z, Zeng J, Chen W, Foo SS, Lee SA, Ge J, Wang S, Goldman SA, Zlokovic BV, et al: Zika virus NS4A and NS4B proteins deregulate Akt-mTOR signaling in human fetal neural stem cells to inhibit neurogenesis and induce autophagy. Cell Stem Cell 19: 663-671, 2016.

19. Li M, Liao YJ, Hou GH, Yang ZB and Zuo ML: Monosialotetrahexosylganglioside protect cerebral ischemia/reperfusion injury through upregulating the expression of tyrosine hydroxylase by inhibiting lipid peroxidation. Biomed Pharmacother 84: 1923-1929, 2016.

20. Hodge RD, Nelson BR, Kahoud RJ, Yang R, Mussar KE, Reiner SL and Hevner RF: Tbr2 is essential for hippocampal lineage progression from neural stem cells to intermediate progenitors and neurons. J Neurosci 32: 6275-6287, 2012.

21. Chen T, Yu Y, Tang LJ, Kong L, Zhang CH, Chu HY, Yin LW and Ma HY: Neural stem cells over-expressing brain-derived neurotrophic factor promote neuronal survival and cytoskeletal protein expression in traumatic brain injury sites. Neural Regen Res 12: 433-439, 2017.

22. Longa EZ, Weinstein PR, Carlson S and Cummins R: Reversible middle cerebral artery occlusion without craniectomy in rats. Stroke 20: 84-91, 1989. 
23. Livak KJ and Schmittgen TD: Analysis of relative gene expression data using real-time quantitative PCR and the 2(-Delta Delta $\mathrm{C}(\mathrm{T})$ ) method. Methods 25: 402-408, 2001

24. Benakis C, Brea D, Caballero S, Faraco G, Moore J, Murphy M, Sita G, Racchumi G, Ling L, Pamer EG, et al: Commensal microbiota affects ischemic stroke outcome by regulating intestinal $\gamma \delta$ T cells. Nat Med 22: 516-523, 2016.

25. Choudhury GR and Ding S: Reactive astrocytes and therapeutic potential in focal ischemic stroke. Neurobiol Dis 85: 234-244, 2016.

26. Imran A, Arshad MU, Arshad MS, Imran M, Saeed F and Sohaib M: Lipid peroxidation diminishing perspective of isolated theaflavins and thearubigins from black tea in arginine induced renal malfunctional rats. Lipids Health Dis 17: 157, 2018.

27. Tipoe GL, Leung TM, Hung MW and Fung ML: Green tea polyphenols as an anti-oxidant and anti-inflammatory agent for cardiovascular protection. Cardiovasc Hematol Disord Drug Targets 7: 135-144, 2007.

28. Fan FY, Sang LX and Jiang M: Catechins and their therapeutic benefits to inflammatory bowel disease. Molecules 22: pii: E484, 2017.

29. Bernatoniene $\mathbf{J}$ and Kopustinskiene DM: The role of catechins in cellular responses to oxidative stress. Molecules 23: pii: E965, 2018.

30. Zhang Y, Xu D, Qi H, Yuan Y, Liu H, Yao S, Yuan S and Zhang J: Enriched environment promotes post-stroke neurogenesis through NF- $\kappa \mathrm{B}$-mediated secretion of IL-17A from astrocytes. Brain Res 1687: 20-31, 2018.

31. Wang J, Fu X, Zhang D, Yu L, Li N, Lu Z, Gao Y, Wang M, Liu X, Zhou C, et al: ChAT-positive neurons participate in subventricular zone neurogenesis after middle cerebral artery occlusion in mice. Behav Brain Res 316: 145-151, 2017.

32. Li P, Shen M, Gao F, Wu J, Zhang J, Teng F and Zhang C: An antagomir to MicroRNA-106b-5p ameliorates cerebral ischemia and reperfusion injury in rats via inhibiting apoptosis and oxidative stress. Mol Neurobiol 54: 2901-2921, 2017.

33. Tao X, Sun X, Xu L, Yin L, Han X, Qi Y, Xu Y, Zhao Y, Wang C and Peng J: Total flavonoids from Rosa laevigata Michx fruit ameliorates hepatic ischemia/reperfusion injury through inhibition of oxidative stress and inflammation in rats. Nutrients 8: pii: E418, 2016.

34. Li K, Ding D and Zhang M: Neuroprotection of osthole against cerebral ischemia/reperfusion injury through an Anti-apoptotic pathway in rats. Biol Pharm Bull 39: 336-342, 2016.

35. Yoshino K, Hara Y, Sano M and Tomita I: Antioxidative effects of black tea theaflavins and thearubigin on lipid peroxidation of rat liver homogenates induced by tert-butyl hydroperoxide. Biol Pharm Bull 17: 146-149, 1994.
36. Han X, Zhang J, Xue X, Zhao Y, Lu L, Cui M, Miao W and Fan S: Theaflavin ameliorates ionizing radiation-induced hematopoietic injury via the NRF2 pathway. Free Radic Biol Med 113: 59-70, 2017.

37. Liu P, Zhao H, Wang R, Wang P, Tao Z, Gao L, Yan F, Liu X, Yu S, Ji X and Luo Y: MicroRNA-424 protects against focal cerebral ischemia and reperfusion injury in mice by suppressing oxidative stress. Stroke 46: 513-519, 2015

38. Zhao H, Tao Z, Wang R, Liu P, Yan F, Li J, Zhang C, Ji X and Luo Y: MicroRNA-23a-3p attenuates oxidative stress injury in a mouse model of focal cerebral ischemia-reperfusion. Brain Res 1592: 65-72, 2014.

39. Liang Y, Xu J, Wang Y, Tang JY, Yang SL, Xiang HG, Wu SX and Li XJ: Inhibition of MiRNA-125b decreases cerebral ischemia/reperfusion injury by targeting CK $2 \alpha / \mathrm{NADPH}$ oxidase signaling. Cell Physiol Biochem 45: 1818-1826, 2018.

40. Zhang R, Liu C, Niu Y, Jing Y, Zhang H, Wang J, Yang J, Zen K, Zhang J, Zhang CY and Li D: MicroRNA-128-3p regulates mitomycin C-induced DNA damage response in lung cancer cells through repressing SPTAN1. Oncotarget 8: 58098-58107, 2016.

41. Chen J, Li W, Li Y, He S, Li L, Liang L, Song Y, Qin D and Zheng H: MicroRNA-128-3p impaired water maze learning by suppressing Doublecortin expression in both wild type and A $\beta-42$ infused mice. Neurosci Lett 626: 79-85, 2016.

42. Chen GH, Xu CS, Zhang J, Li Q, Cui HH, Li XD, Chang LP, Tang RJ, Xu JY, Tian XQ, et al: Inhibition of miR-128-3p by tongxinluo protects human cardiomyocytes from Ischemia/reperfusion injury via Upregulation of p70s6k1/p-p70s6k1. Front Pharmacol 8: 775, 2017.

43. Zhou J, He Z, Guo L, Zeng J, Liang P, Ren L, Zhang M, Zhang P and Huang X: MiR-128-3p directly targets VEGFC/VEGFR3 to modulate the proliferation of lymphatic endothelial cells through $\mathrm{Ca}^{2+}$ signaling. Int J Biochem Cell Biol 102: 51-58, 2018.

44. Mao G, Ren P, Wang G, Yan F and Zhang Y: MicroRNA-128-3p protects mouse against cerebral ischemia through reducing $\mathrm{p} 38 \mathrm{a}$ mitogen-activated protein kinase activity. J Mol Neurosci 61: 152-158, 2017.

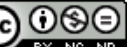

This work is licensed under a Creative Commons Attribution-NonCommercial-NoDerivatives 4.0 International (CC BY-NC-ND 4.0) License. 